\title{
25 Research Suare \\ The Value of Colorectal Filling Contrast Ultrasonography in Diagnosing Paediatric Juvenile Polyps
}

\section{Min Zhang}

Hainan General Hospital

Hai Lin

Hainan General Hospital

Tang Na Wu

Hainan General Hospital

Jie Hu

Hainan General Hospital

Sheng Xin Fu

Hainan General Hospital

Xiang Xiang Jing

Hainan General Hospital

Ling Ling Qin ( $\nabla$ qinlingling2327@163.com )

Hainan General Hospital

\section{Research Article}

Keywords: Colorectal filling contrast ultrasonography, juvenile polyps, paediatric, gastrointestinal

Posted Date: August 5th, 2021

DOI: https://doi.org/10.21203/rs.3.rs-744642/v1

License: (9) This work is licensed under a Creative Commons Attribution 4.0 International License.

Read Full License 


\section{Abstract}

Objectives: To describe a facilitated procedure of colorectal filling contrast ultrasonography (CFCUS) and investigate its potential role in the assessment of paediatric juvenile polyps.

Methods: The general information, clinical symptoms, ultrasound image characteristics, and colonoscopy findings of 101 children with clinical signs of colorectal polyps admitted to our hospital between May 2018 and May 2021 were retrospectively reviewed. All children underwent conventional transabdominal ultrasonography (CTUS), and CFCUS before colonoscopy, and the latter served as the gold standard. Chisquared tests and Mann-Whitney $U$ tests were used for the statistical analysis.

Results: Forty-five children with fifty-two colorectal polyps were diagnosed by colonoscopy. Ten polyps had false-negative results on CTUS, six of which were detected by CFCUS. The sensitivity, specificity, positive predictive value, and negative predictive value of CFCUS vs CTUS was $92.3 \%$ vs $80.7 \%, 100 \%$ vs $100 \%, 100 \%$ vs $100 \%, 93.3 \%$ vs $84.8 \%$, respectively. The maximum diameter of polyps (mean \pm standard deviation) missed by CTUS was significantly smaller than that detected by CTUS $(7.50 \pm 2.12 \mathrm{~mm}$ [range 4-12 mm] vs. $19.62 \pm 7.89 \mathrm{~mm}$ [range $6-41 \mathrm{~mm}$ ], $\mathrm{P}<0.0001)$. A significant difference was observed between CTUS and CFCUS. The difference between CTUS and CFCUS regarding the detection rate of polyps with a diameter $<1 \mathrm{~cm}(P=0.031)$. A statistical difference was observed in the detection rate of polyps $(P=0.031)$, and pedicles $(P<0.001)$ between CTUS and CFCUS. The Kappa values for the assessment of Yamada's classification between CTUS and colonoscopy and CFCUS and colonoscopy were 0.51 and 0.84 , respectively. Six colonic polyps were accompanied by a punctate hyperechoic area on the surface, which may be suggestive of a correlation with erosion and bleeding findings.

Conclusion: CFCUS can increase the detection rate of polyps and pedicles, especially polyps with diameters $<1 \mathrm{~cm}$, and accurately evaluate Yamada's classification, providing useful preoperative information for colonoscopy.

\section{Introduction}

Gastrointestinal polyps which have a prevalence of $1-2 \%$ in preschool and school-age children, are the most common cause of painless rectal bleeding [1]. Based on the World Health Organization (WHO) classification, polyps can be divided into adenomas, hyperplastic, inflammatory and hamartomatous. The most common pathological type of polyps in paediatric patients is hamartomas, the majority of which are benign and located in the colon. Juvenile polyps are considered to be a type of hamartomatous polyp, which accounts for a predominant proportion of polyps in children. Juvenile polyps are charactered by fewer than five hamartomatous polyps without extraintestinal manifestations or a positive family history[2]. Four morphological subgroups of polyps can be further described according to Yamada's classification: type l: a mound-shaped polyp with a raised part that is smooth without a clear boundary; type II: a hemispherical shaped sessile polyp with an uplifted part that has a definite boundary; type III: a polyp with an uplift that is slightly smaller, forming a stubby pedicle; and type IV: a polyp that 
has an obvious pedicle[3, 4]. Previous studies have demonstrated that polyps with diameters $>10 \mathrm{~mm}$ and those classified as Yamada type III are considered risk factors associated with bleeding after the endoscopic resection of colorectal polyps with delayed bleeding $[2,5]$.

Colonoscopy is the gold standard for the histological evaluation of juvenile polyps and serves as the primary method for the resection of polyps. The invasiveness of colonoscopy makes its application limited as a first-line diagnostic tool in the diagnosis of polyps. With advanced ultrasound techniques for paediatric imaging, ultrasound examination observes the general features of the colonic polyps, including the location in the colonic lumen, size, and blood flow in polyps. The typical sonographic features of juvenile polyps are spherical or ovoid hypoechoic nodules in the colon, some of which may contain small cysts inside. An effective preoperative diagnosis can not only reduce negative detection by colonoscopy, but also minimize intraoperative complications and anaesthetic risks. However, factors of obesity, concealment by gas and/or stool, and ultrasonographic blind regions of the colon may be associated with misdiagnosis on ultrasound[6]. Many investigations on sonographic techniques such as grade compression, segment approach and hydrocolonic sonography have beenconducted in regard to advancing the detection rate of polyps[7-11].

The purpose of this study was to investigate a modified, facilitated procedure of colorectal filling contrast ultrasonography (CFCUS) and compare it to conventional transabdominal ultrasonography (CTUS) in the assessment of polyps in children. CFCUS plays an important role in selecting examination and treatment modalities for a child suspected of having gastrointestinal polyps.

\section{Materials And Methods}

\section{Patients}

The study protocol was approved by the institutional review board, and informed consent was obtained from children's guardians. The inclusion criteria were as follows: children with polyp-suggestive symptoms, such as haematochezia, abdominal pain, recurrent intussusception and diarrhoea, who were recommended to undergo CTUS and CFCUS before colonoscopy from May 2018 to May 2021 at our institution. The related medical records spanning a 3-year period were reviewed. A total of 101 children ( 69 boys and 42 girls with a mean \pm standard deviation age of $5.47 \pm 2.18$ years and an age range of 1.0 to 11.6 years met the criteria for inclusion. The exclusion criteria were as follows: children suspected of having bowel obstruction, perforation, or peritonitis; children with colonoscopy performed outside our hospital or performed before ultrasound in our hospital; children younger than 1 year of age; children without CFCUS date; and children suspected of having polyposis syndrome. The study was approved by the institutional review board of our hospital (Med-Eth-Re 2020191). Prior to the study, informed consent was obtained from each participant or their guardian (for underaged participants).

\section{Equipment}


A HITACHI VISION Ascendus scanner (Hitachi Manufacturing Co, Ltd., Chiba Prefecture, Japan) equipped with an L12-5 linear-array transducer (5-12 MHz) and a C8-4 small convex-array transducer (4-8 $\mathrm{MHz})$ was used for the ultrasound examinations in this study.

\section{Review process}

Two ultrasound specialists (ZM, HJ) who each had 6 years of clinical experience in paediatric gastrointestinal ultrasound diagnosis retrospectively reviewed all the images by consensus. During the image review, the specialists were blinded to the definitive diagnosis of colonoscopy, other imaging information and the medical records. The inspection process of CTUS and CFCUS was mutually blinded.

\section{Preparation}

The children did not need to fast before the ultrasound examination, but at least 30 minutes before their examination, a glycerine enema (10-30 ml) was performed to clean the colon from the rectum to the ascending colon as much as possible. Children who could not cooperate were given a $10 \%$ chloral hydrate $(0.5 \mathrm{ml} / \mathrm{kg})$ oral dose with parental consent before the examination.

Southern brand black sesame paste ${ }^{\circledR}$ (Guangxi, China) was used as an echo luminal contrast agent (40 g per package); it is composed of rice, black sesame, walnut, peanut and maltodextrin. Forty grams of powder was mixed and gently stirred with $300 \mathrm{ml}$ of $80^{\circ} \mathrm{C}$ water to form a homogeneous suspension, which could provide an echo window and eliminate gas and/or food debris The suspension was allowed to $\mathrm{cool}$ to $35^{\circ} \mathrm{C}$ to $38^{\circ} \mathrm{C}$ before instillation into the rectum through nasogastric tube (Fig .1).

\section{Ultrasound protocol}

Ultrasound examinations were performed before colonoscopy. The examinations were conducted by four specialists who had (10 years (QLL),8 years (WTN), 7 years (ZM), and 7 years (HJ)) of clinical experience in the diagnosis of paediatric gastrointestinal ultrasonography. Children were placed in the supine position with adequate exposure of the abdomen. Various parameters were adjusted to the best level; mechanical index (MI) was 0.6-0.8, Tis was lower than 0.4, the dynamic range was 50-70 dB, and the depth was $8 \mathrm{~cm}$. The focus was placed at a depth of $2-4 \mathrm{~cm}$, and the length of the focus was $1 \mathrm{~cm}$. The CTUS examination followed this sequence: rectum, sigmoid colon, descending colon, transverse colon, ascending colon, and ileocecal valve. Longitudinal and axial images were acquired for each section of the colon. Once suspicious polyps were detected, the location, morphology, size, distribution of blood flow, and Yamada classification were acquired. The dynamic imaging of the entire CFCUS process was stored on a hard disk.

A 12-Fr nasogastric tube connected to a $50 \mathrm{ml}$ syringe lubricated with paraffin oil was inserted into the rectum through the anus. A total of $200-1000 \mathrm{ml}$ echo intestinal filling agent (depending on the distention condition of the large intestine) was injected with a speed of $50 \mathrm{ml} / \mathrm{min}$ by a nurse. CFCUS was performed immediately when the large intestine was satisfactorily filled with the echo intestinal filling agent. Children were asked to take a right lateral decubitus position to make the intestinal filling 
agent flow into the transverse or right colon when needed. The entire CFCUS procedure lasted from 15 to 30 minutes, and the sequence was the same as that of CTUS.

\section{Statistical analysis}

The sensitivity, specificity, and positive and negative predictive values of CFCUS and CTUS based on the results of colonoscopy as the gold standard and the mean \pm standard deviation were used to present quantitative data. Statistical differences in the detection rates of polyps and pedicles were compared using the Chi-squared test, and the differences in the sizes of polyps missed and detected were compared using the Mann-Whitney U test. SPSS software (Version 25.0, Chicago, IL) was used for the statistical analysis. Date was considered significant if the $p$-value $<0.05$. The Kappa value was used to evaluate the agreement between CTUS and colonoscopy vs CFCUS and colonoscopy, with the interpretation of poor agreement $\leq 0.40$, moderate agreement $0.4-0.75$, and excellent agreement $\geq 0.75$.

\section{Results}

\section{Clinical manifestations and pathological results}

Forty-five children with fifty-two colorectal polyps were diagnosed by colonoscopy, and the mean age was $4.87 \pm 1.95$ years (range 1.7-10.2 years). The mean diameter of the polyps was $17.28 \pm 7.88 \mathrm{~mm}$ (range 4-41 mm). The histopathological diagnosis of all colorectal polyps was juvenile polyps. In the 56 children without polyps, the colonoscopy results were as follows: normal findings, $n=29$; nonspecific colitis, $n=12$; anal fissure, $n=7$; ulcerative colitis, $n=4$; Crohn's disease, $n=3$; and eosinophilic colitis, $n=$ 1. The general information of the children with juvenile polyps is shown in Table 1. 
Table 1

Clinical presentation and postoperative findings in children with juvenile polyps

\begin{tabular}{|c|c|c|}
\hline & $\mathbf{N}$ & $\%$ \\
\hline \multicolumn{3}{|l|}{ Sex } \\
\hline Male & 29 & 64.4 \\
\hline Female & 16 & 35.6 \\
\hline \multicolumn{3}{|l|}{ Clinical manifestations } \\
\hline Rectal bleeding & 34 & 75.6 \\
\hline Abdominal pain & 9 & 20.0 \\
\hline Prolapse of the polyp from the anus & 4 & 8.9 \\
\hline Diarrhea & 2 & 4.4 \\
\hline \multicolumn{3}{|l|}{ Solitary or Multiple polyp } \\
\hline Solitary polyp & 41 & 91.1 \\
\hline Multiple polyps (the number of polyps $\geq 2$ ) & 4 & 8.9 \\
\hline \multicolumn{3}{|l|}{ Location } \\
\hline Cecum & 2 & 3.8 \\
\hline Ascending colon & 3 & 5.7 \\
\hline Transverse colon & 3 & 5.7 \\
\hline Descending colon & 4 & 7.7 \\
\hline Sigmoid colon & 28 & 53.8 \\
\hline Rectum & 12 & 23.1 \\
\hline \multicolumn{3}{|l|}{ diameter of the polyp } \\
\hline$<1 \mathrm{~cm}$ & 14 & 26.9 \\
\hline$<1 \mathrm{~cm}$ & 18 & 34.6 \\
\hline$<2 \mathrm{~cm}$ and $\geq 1 \mathrm{~cm}$ & 15 & 28.8 \\
\hline$<3 \mathrm{~cm}$ and $\geq 2 \mathrm{~cm}$ & 5 & 9.6 \\
\hline \multicolumn{3}{|l|}{$\geq 3 \mathrm{~cm}$} \\
\hline Type I & 0 & 0 \\
\hline Type II & 0 & 0 \\
\hline
\end{tabular}




\begin{tabular}{|lll|}
\hline & N & $\%$ \\
\hline Type III & 3 & 5.8 \\
\hline Type IV & 49 & 94.2 \\
\hline
\end{tabular}

\section{Diagnostic ultrasound results}

Ten polyps had false-negative results on CTUS, six of which were detected by CFCUS (Table 2 and Fig. 2). The sensitivity, specificity, positive predictive value, and negative predictive value of CFCUS vs CTUS was $92.3 \%$ (48 of 52 ) vs $80.7 \%$ (42 of 52 ), $100 \%$ ( 56 of 56 ) vs $100 \%$ (56 of 56 ), $100 \%$ (48 of 48 ) vs $100 \%$ (42 of 42 ), $93.3 \%$ ( 56 of 60 ) vs $84.8 \%$ ( 56 of 66 ), respectively. The maximum diameter of polyps (mean \pm standard deviation) missed by CTUS was significantly smaller than that detected by CTUS $(7.50 \pm 0.67$ $\mathrm{mm}$ [range 4-12 mm] vs. $21.93 \pm 1.45 \mathrm{~mm}$ [range 6-41 mm], $\mathrm{P}<0.0001$ ) (Fig. 3). A significant difference was observed between CTUS and CFCUS regarding the detection rate of polyps $(P=0.031)$. In 52 colorectal polyps, 14 polyps had a diameter $<1 \mathrm{~cm}$ and 38 polyps had a diameter $\geq 1 \mathrm{~cm}$. A significant difference was observed between CTUS and CFCUS regarding the detection rate of polyps with a diameter $<1 \mathrm{~cm}(P=0.031)$. No significant difference was observed between CTUS and CFCUS regarding the detection rate of polyps with a diameter $\geq 1 \mathrm{~cm}(P=1.000)$. 
Table 2

The characteristics of ten polyps missed by ultrasound

\begin{tabular}{|c|c|c|c|c|c|c|c|}
\hline No. & Gender & $\begin{array}{l}\text { Age } \\
\text { (years) }\end{array}$ & $\begin{array}{l}\text { Solitary or } \\
\text { Multiple } \\
\text { polyps }\end{array}$ & $\begin{array}{l}\text { Size of } \\
\text { polyps } \\
(\mathrm{mm})\end{array}$ & $\begin{array}{l}\text { Location by } \\
\text { colonoscopy }\end{array}$ & $\begin{array}{l}\text { detected } \\
\text { by CTUS }\end{array}$ & $\begin{array}{l}\text { detected } \\
\text { by CFCUS }\end{array}$ \\
\hline 1 & $M$ & 8.7 & Solitary & $7 \times 6$ & Rectum & $\begin{array}{l}\text { No } \\
\text { detected }\end{array}$ & Rectum \\
\hline 2 & $M$ & 3.3 & Solitary & $8 \times 5$ & $\begin{array}{l}\text { Sigmoid } \\
\text { colon }\end{array}$ & $\begin{array}{l}\text { No } \\
\text { detected }\end{array}$ & $\begin{array}{l}\text { Sigmoid } \\
\text { colon }\end{array}$ \\
\hline 3 & $M$ & 11.7 & Solitary & $12 \times 8$ & $\begin{array}{l}\text { Ascending } \\
\text { colon }\end{array}$ & $\begin{array}{l}\text { No } \\
\text { detected }\end{array}$ & $\begin{array}{l}\text { No } \\
\text { detected }\end{array}$ \\
\hline 4 & $\mathrm{~F}$ & 3.9 & Solitary & $8 \times 5$ & Rectum & $\begin{array}{l}\text { No } \\
\text { detected }\end{array}$ & Rectum \\
\hline 5 & $M$ & 5.4 & Solitary & $9 \times 8$ & Rectum & $\begin{array}{l}\text { No } \\
\text { detected }\end{array}$ & Rectum \\
\hline 6 & $\mathrm{~F}$ & 7.0 & Solitary & $6 \times 5$ & $\begin{array}{l}\text { Sigmoid } \\
\text { colon }\end{array}$ & $\begin{array}{l}\text { No } \\
\text { detected }\end{array}$ & $\begin{array}{l}\text { Sigmoid } \\
\text { colon }\end{array}$ \\
\hline 7 & $M$ & 3.6 & Multiple & $8 \times 7$ & $\begin{array}{l}\text { Sigmoid } \\
\text { colon }\end{array}$ & $\begin{array}{l}\text { No } \\
\text { detected }\end{array}$ & $\begin{array}{l}\text { Sigmoid } \\
\text { colon }\end{array}$ \\
\hline 8 & $\mathrm{~F}$ & 2.6 & Multiple & $6 \times 4$ & $\begin{array}{l}\text { Sigmoid } \\
\text { colon }\end{array}$ & $\begin{array}{l}\text { No } \\
\text { detected }\end{array}$ & $\begin{array}{l}\text { No } \\
\text { detected }\end{array}$ \\
\hline 9 & $\mathrm{~F}$ & 4.3 & Solitary & $7 \times 6$ & Rectum & $\begin{array}{l}\text { No } \\
\text { detected }\end{array}$ & $\begin{array}{l}\text { No } \\
\text { detected }\end{array}$ \\
\hline 10 & $M$ & 4.8 & Multiple & $8 \times 4$ & $\begin{array}{l}\text { Transverse } \\
\text { colon }\end{array}$ & $\begin{array}{l}\text { No } \\
\text { detected }\end{array}$ & $\begin{array}{l}\text { No } \\
\text { detected }\end{array}$ \\
\hline
\end{tabular}

\section{Ultrasound image characteristics of polyps}

Forty-eight polyps displayed a spherical or ovoid hypoechoic nodule within the colonic lumen by CFCUS. Forty-two polyps showed a heterogeneous echo, and six polyps showed a uniform medium echo. Among the 48 juvenile polyps, small cysts were detected in 38 polyps (73.1\%).

Thirty-one pedicles (64.6\%) connected to the polyps and the colonic inner wall were detected by CTUS, while 48 pedicles (100\%) were detected by CFCUS. Among the 52 polyps confirmed by colonoscopy, 3 polyps were diagnosed as Yamada type III and 49 were diagnosed as Yamada type IV. The Kappa value for the assessment of Yamada's classification between CTUS and colonoscopy vs CFCUS and colonoscopy was 0.51 vs 0.84 (Tables $3-4$ ), demonstrating excellent agreement between CFCUS and colonoscopy ( $\geq 0.75$ ). A significant difference was observed in the detection rate of the pedicle of the polyp between CTUS and CFCUS $(P<0.001)$. Different images of polyps detected by CTUS and CFCUS 
were obtained (Fig. 4-6). CFCUS revealed a punctate hyperechoic area on the surface in six colonic polyps, and all displayed erosion and bleeding during colonoscopy (Fig. 7). Four solitary polyp was detected in the head of the intussusception by routine transabdominal ultrasonography (Fig. 8).

Table 3

The assessment of Yamada classified type between CTUS and colonoscopy

\begin{tabular}{|llll|}
\hline Colonoscopy & CTUS & total \\
\hline & Yamada classification III & Yamada classification IV & \\
\hline Yamada classification III & 2 & 1 & 3 \\
\hline Yamada classification IV & 2 & 26 & 28 \\
\hline Total & 4 & 27 & 31 \\
\hline Kappa $=0.518$ & & & \\
\hline
\end{tabular}

Table 4

The assessment of Yamada classified type between CFCUS and colonoscopy

\begin{tabular}{|lllc|}
\hline Colonoscopy & CFUCS & total \\
\hline & Yamada classification III & Yamada classification IV & \\
\hline Yamada classification III & 3 & 0 & 3 \\
\hline Yamada classification IV & 1 & 44 & 45 \\
\hline Total & 4 & 44 & 48 \\
\hline Kappa $=0.846$ & & & \\
\hline
\end{tabular}

\section{Discussion}

Juvenile polyps are the most common pathological type of colonic polyps in children. The most frequent clinical manifestation is rectal bleeding, the incidence rate of which was $75.6 \%$ in our study. Chronic haemorrhage caused by polyps may result in anaemia and larger polyps may result in secondary intussusception or intestinal obstruction. Currently, high-frequency sonography has been widely applied to diagnose colonic polyps in children. Due to the disturbing effects of gastrointestinal luminal contents, conventional transabdominal sonography does not easily visualize intestinal lesions. Sonographic evaluations of the colon using water or a gastrointestinal echo intestinal filling agent, known as hydrocolonic sonography or contrast/filling intestinal ultrasonography, have been proposed as an 
alternative to barium enema and colonoscopy for diagnosing and staging gastrointestinal lesions in adults[12-17]. Previous clinical studies have demonstrated that compared with conventional sonography, the application of gastrointestinal filling agents in sonographic examinations of the bowel provides a reliable evaluation and improves the detection of lesions in the gastrointestinal tract[18, 19]. To our knowledge, the role of filling sonography in children with gastrointestinal polyps has not been evaluated.

In this article, Southern brand black sesame paste ${ }^{\circledR}$ (Guangxi, China) was used as gastrointestinal intraluminal filling agent, it is a safe, inexpensive, food-grade product that is well tolerated by virtually all children older than 1 year. The gastrointestinal intraluminal filling agent can eliminate the interference of gas and mucus in the lumen, producing a clear contrast effect with the surrounding tissues. Moreover, because the application of a tube with balloon prevents the simultaneous visualization of the lower rectum, simultaneously, for the purpose of minimizing suffering and relieving pain in children, a 12-Fr nasogastric tube was used. In this way, modified CFCUS. can not only improve the detection rate of polyps and pedicles but also optimize the morphological assessment by offering better visualization of the intestinal cavity, especially the lower rectum.

The maximum diameter of polyps missed by CTUS was significantly smaller than that detected by CTUS, $(P<0.0001)$. Compared to CTUS, CFCUS increased the detection rate and reduced the misdiagnosis of polyps, especially those with diameters $<1 \mathrm{~cm}(P<0.05)$. The potential reasons for missed diagnosis were considered to be related to the sonographer 's focus on larger polyps and neglect of small ones, or the Inadequate intestinal preparation which may lead to unclear visualization of the colon. In addition, colon anatomical variant such as redundant sigmoid colon/ malrotation of intestine et al, might be another reason for false-negative results.

Previous studies have shown that the morphology of polyp is one of an important factor related to the complications of a colonoscopy resection of polyps[20]. Examples include Yamada type III polyps (those with short pedicles), regarding mucosal burns and perforation during resection; large polyps that contain large blood vessels in the pedicle that can cause bleeding after the endoscopic resection of colorectal polyps; and delayed bleeding $[5,20]$. Therefore, the pedicle of polyps needed to be evaluated in detail before colonoscopy for accurate evaluations of Yamada classified. However, inhomogeneous air content and an unfilled intestinal cavity make it difficult to accurately distinguish the pedicle from normal mucosa by CTUS. CFCUS was performed in the filling state of the intestinal cavity, which can avoid the excessive pulling of the intestinal wall mucosa by the pedicle. In this study, CFCUS increased the detection rate of the pedicle $(\mathrm{P}<0.001)$ and reduced the overestimation of the pedicle, demonstrating excellent agreement in the Yamada classification between CFCUS and colonoscopy (Kappa value $=$ 0.846), and providing adequate evaluation for surgical treatment.

Thirty-eight juvenile polyps exhibited a hypoechoic nodule in the colon lumen, accompanied by small cystic areas within it. Juvenile polyps are derived from the mucosal epithelium and present mild to moderate gland hyperplasia in pathology. Glandular epithelial differentiation and manifestation of 
glandular expansion are associated with cysts in polyps on a sonogram. Another incidental finding was punctate hyperechoic attachments on the surface in six polyps by CFCUS, revealing erosion and bleeding on the polyp surface by colonoscopy. The imaging features of ultrasound may be suggestive of a correlation with active bleeding finding, however, it could also be due to artefacts or trapped gas bubbles. Further validation is needed with a large sample, multicentre clinical trial.

The shortcomings of this study are as follows: this was a single-centre, retrospective study, using a single pathological type, and the sample size was relatively small. Future prospective studies on a large number of children with polyps and an in-depth study on pedicles are required.

\section{Conclusions}

CFCUS is a convenient and inexpensive procedure for the detection of colonic polyps in children. Compared to CTUS, CFCUS not only increases the detection rates of polyps and the pedicles, but also accurately evaluate Yamada's classification, providing useful preoperative information for colonoscopy.

\section{Abbreviations}

CFCUS colorectal filling contrast ultrasonography

CTUS conventional transabdominal ultrasonography

\section{Declarations}

\section{Ethics approval and consent to participate}

The study has been approved by the ethics committee of Hainan General Hospital, ethical approval NO.: Med- Eth-Re [2020] 195.The authors declare that written informed consent was obtained from a parent or guardian for participants.

\section{Consent for publication}

Not applicable

\section{Availability of data and material}

The datasets used and/or analysed during the current study are available from the corresponding author on reasonable request.

\section{Competing interests}

The authors declare that they have no conflicts of interest.

\section{Funding}


No funding was obtained for this study.

\section{Authors' contributions}

LLQ and MZ designed the study, conducted the literature review, analysed the data, and drafted the manuscript. JH and TNW created the figures. XXJ contributed to drafting the manuscript. HI and SXF contributed to designing the study and drafting the manuscript. All authors read and approved the final manuscript. All authors have read and approved the manuscript", and ensure that this is the case.

\section{Acknowledgements}

Not applicable

\section{Statement}

All methods were carried out in accordance with relevant guidelines and regulations.

\section{Publisher's note}

Springer Nature remains neutral with regard to jurisdictional claims in published maps and institutional affiliations.

\section{References}

1. Bhatia A, Saxena AK, Kalra N, Sodhi KS, Thapa BR, Rao KL, Khandelwal N. Intravenous contrast enhanced computed tomography colonoscopy in children with suspected colonic polyps. Eur $\mathrm{J}$ Radiol. 2013;82(6):905-12.

2. Kay M, Eng K, Wyllie R. Colonic polyps and polyposis syndromes in pediatric patients. Curr Opin Pediatr. 2015;27(5):634-41.

3. Yu L, Li N, Zhang XM, Wang T, Chen W. Analysis of 234 cases of colorectal polyps treated by endoscopic mucosal resection. World J Clin Cases. 2020;8(21):5180-7.

4. Kjølhede T, Ølholm AM, Kaalby L, Kidholm K, Qvist N, Baatrup G. Diagnostic accuracy of capsule endoscopy compared with colonoscopy for polyp detection: systematic review and meta-analyses. Endoscopy. 2020.

5. Park SK, Seo JY, Lee MG, et al. Prospective analysis of delayed colorectal post-polypectomy bleeding. Surg Endosc. 2018;32(7):3282-9.

6. Kuzmich S, Harvey CJ, Kuzmich T, Tan KL. Ultrasound detection of colonic polyps: perspective. $\mathrm{Br} \mathrm{J}$ Radiol. 2012;85(1019):e1155-64.

7. Parra DA, Navarro OM. Sonographic diagnosis of intestinal polyps in children. Pediatr Radiol. 2008;38(6):680-4.

8. Baldisserotto M, Spolidoro JV, Bahú Mda G. Graded compression sonography of the colon in the diagnosis of polyps in pediatric patients. AJR Am J Roentgenol. 2002;179(1):201-5. 
9. Qu NN, Liu RH, Shi L, Cao XL, Yang YJ, Li J. Sonographic diagnosis of colorectal polyps in children: Diagnostic accuracy and multi-factor combination evaluation. Medicine (Baltimore). 2018;97(39):e12562.

10. Maconi G, Radice E, Bareggi E, Porro GB. Hydrosonography of the gastrointestinal tract. AJR Am J Roentgenol. 2009;193(3):700-8.

11. Wang Y, Wang XM, Jia LQ. Ultrasonography with the colonic segment-approach for colonic polyps in children. Pediatr Radiol. 2019;49(13):1735-41.

12. Xu L, Wang X, Wu W, Li Y. Diagnostic Accuracy of Double Contrast-Enhanced Ultrasonography in Clarifying Tumor Depth (T Stage) of Gastric Cancer: Meta-analysis. Ultrasound Med Biol. 2021.

13. Wang L, Liu Z, Kou H, He H, Zheng B, Zhou L, Yang Y. Double Contrast-Enhanced Ultrasonography in Preoperative T Staging of Gastric Cancer: A Comparison With Endoscopic Ultrasonography. Front Oncol. 2019;9:66.

14. Pan M, Huang P, Li S, Chen J, Wei S, Zhang Y. Double contrast-enhanced ultrasonography in preoperative Borrmann classification of advanced gastric carcinoma: comparison with histopathology. Sci Rep. 2013;3:3338.

15. Shi H, Yu XH, Guo XZ, et al. Double contrast-enhanced two-dimensional and three-dimensional ultrasonography for evaluation of gastric lesions. World J Gastroenterol. 2012;18(31):4136-44.

16. Huang P, Li S, Aronow WS, et al. Double contrast-enhanced ultrasonography evaluation of preoperative Lauren classification of advanced gastric carcinoma. Arch Med Sci. 2011;7(2):287-93.

17. Zheng Z, Yu Y, Lu M, et al. Double contrast-enhanced ultrasonography for the preoperative evaluation of gastric cancer: a comparison to endoscopic ultrasonography with respect to histopathology. Am J Surg. 2011;202(5):605-11.

18. Limberg B. Diagnosis of large bowel tumours by colonic sonography. Lancet. 1990;335(8682):144-6.

19. Dixit R, Chowdhury V, Kumar N. Hydrocolonic sonography in the evaluation of colonic lesions. Abdom Imaging. 1999;24(5):497-505.

20. Soh JS, Seo M, Kim KJ. Prophylactic clip application for large pedunculated polyps before snare polypectomy may decrease immediate postpolypectomy bleeding. BMC Gastroenterol. 2020;20(1):68.

\section{Figures}




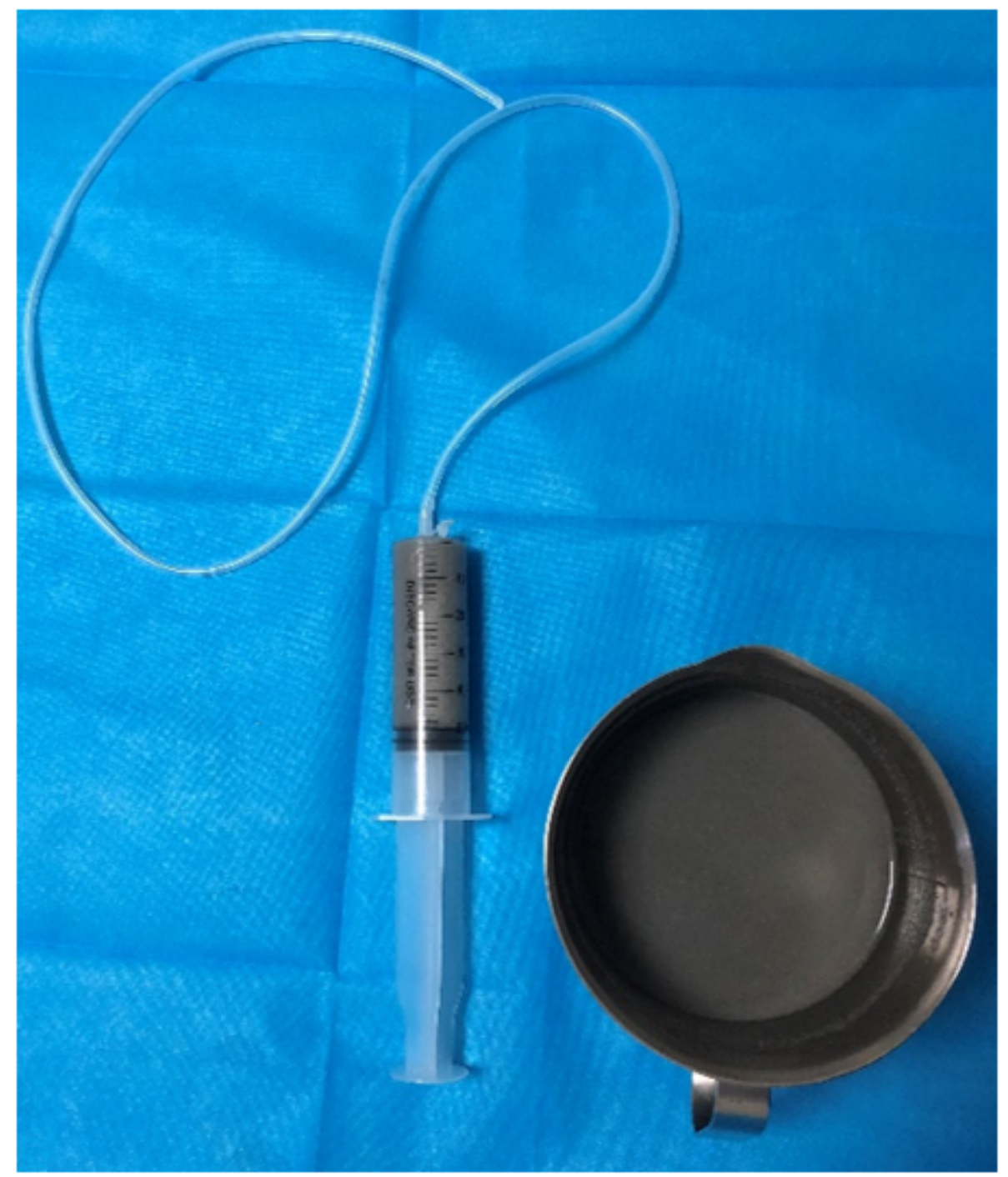

\section{Figure 1}

Photograph of the preparation before CFCUS. A 12-Fr nasogastric tube connected to a $50 \mathrm{ml}$ syringe was lubricated, and Southern brand black sesame paste ${ }^{\circledR}$ (Guangxi, China) was used as the echo luminal contrast agent. 


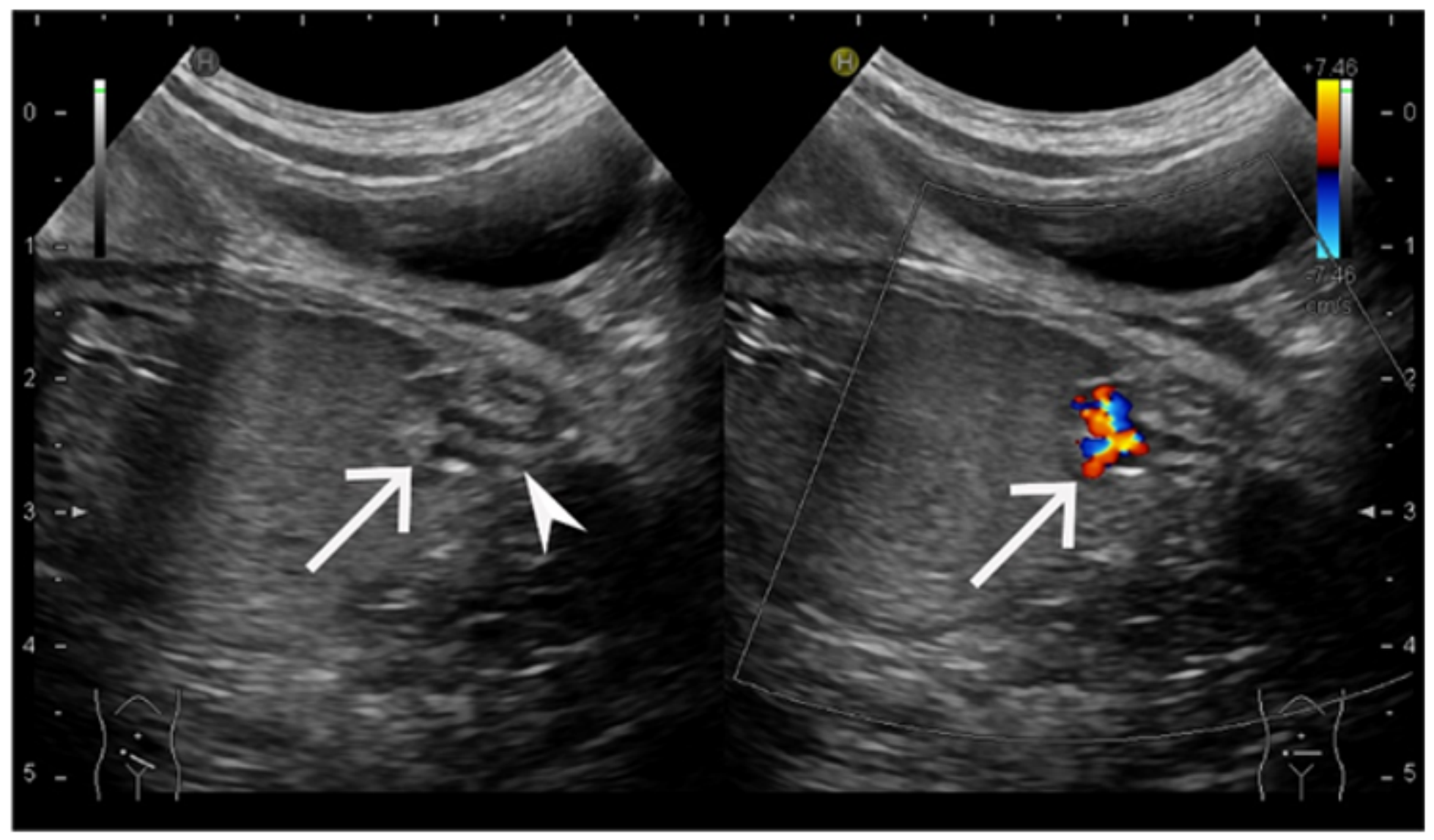

\section{Figure 2}

A 4-year-old boy with a juvenile polyp with false-negative results on CTUS. A small polyp (arrow) with a pedicle (arrowhead) was detected by CFCUS. Colour Doppler demonstrated abundant blood flow signals within the polyp (arrows). 


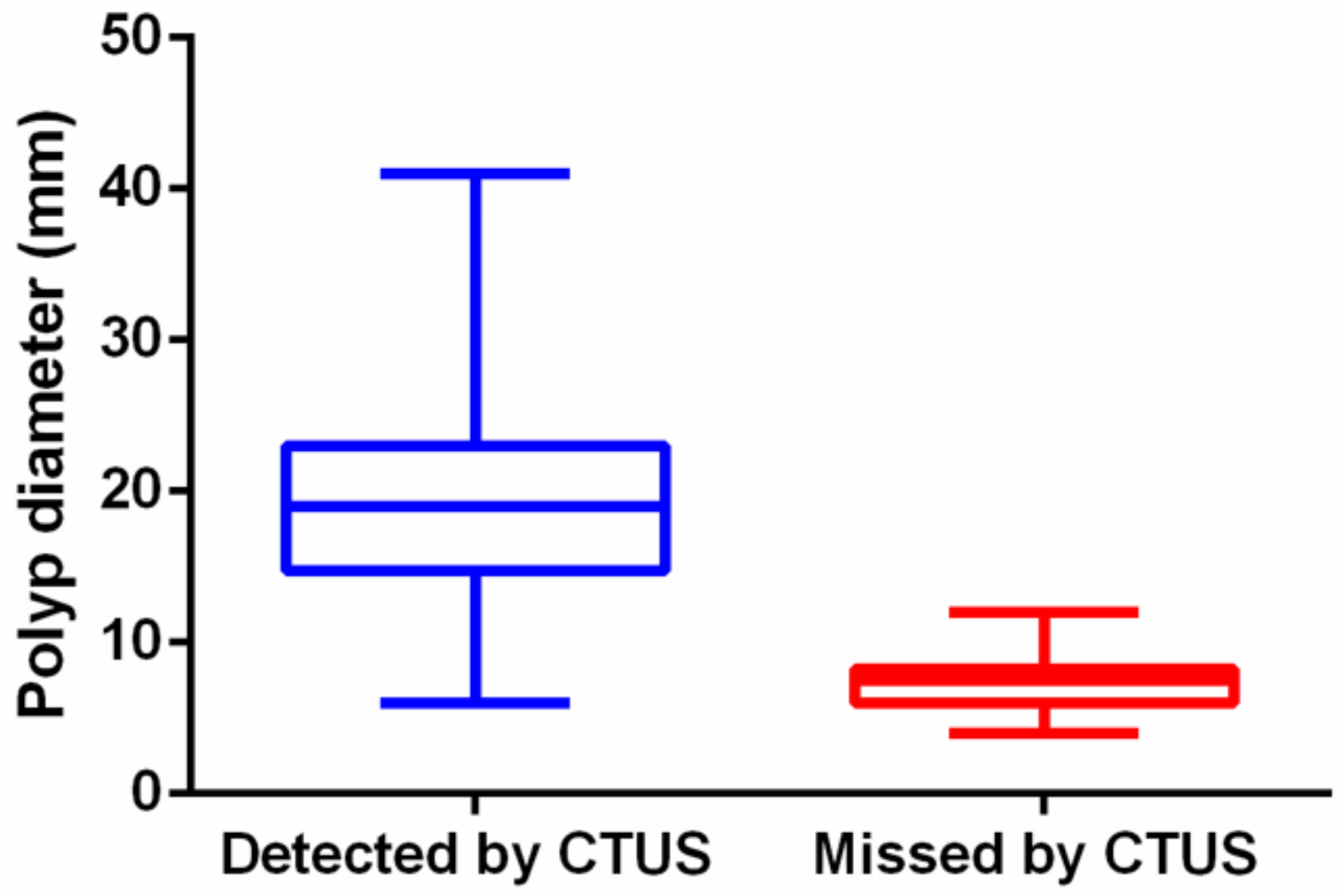

Figure 3

The diameter distribution of polyp between missed groups and detected groups by CTUS.
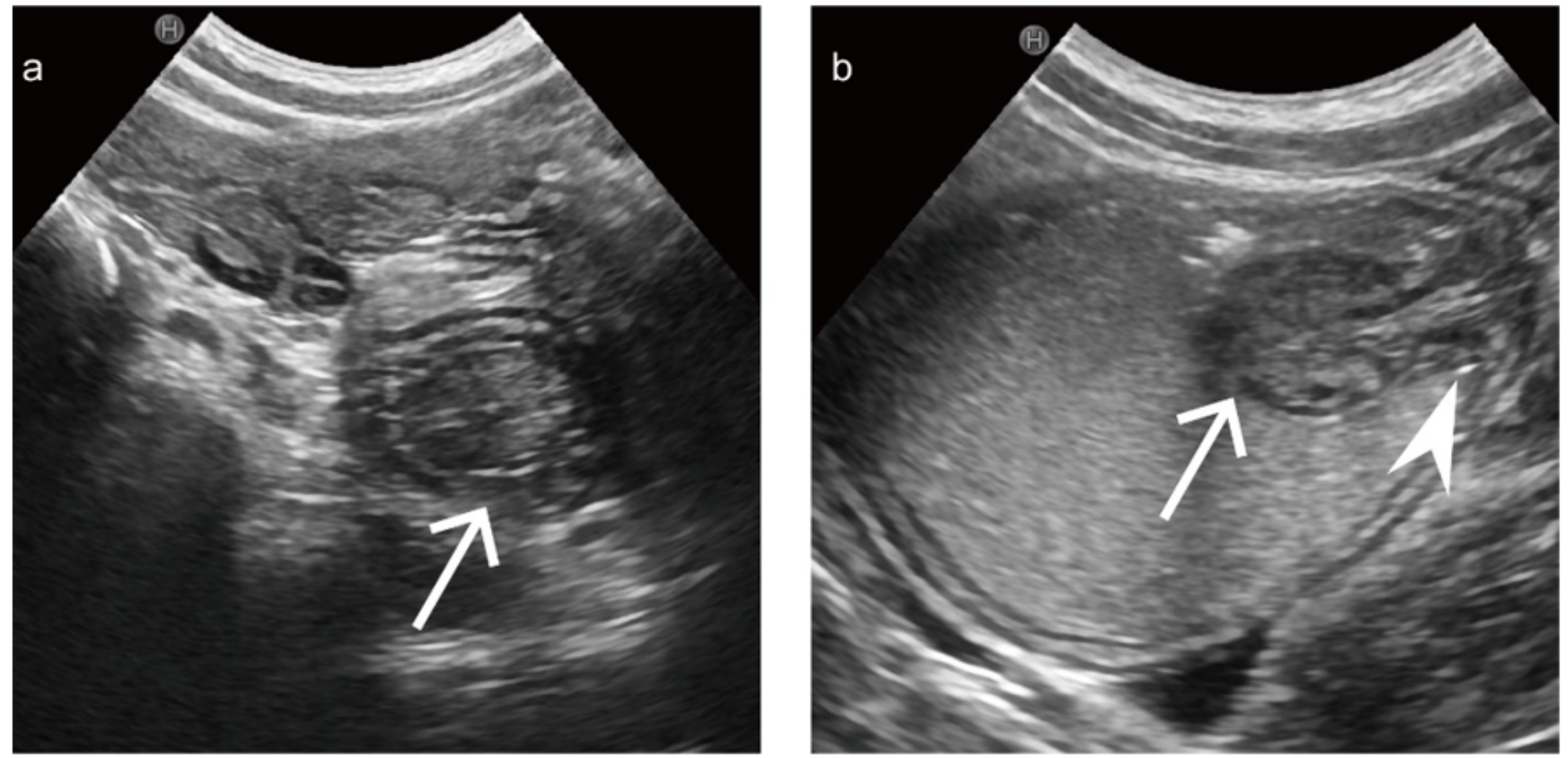


\section{Figure 4}

A 2-year-old boy. Sonography of a polyp located in the rectum was obtained using different ultrasonography techniques. (a) CTUS showed an ovoid hypoechoic nodule (arrow) within the rectum, while the pedicle could not be displayed clearly. (b) Images of the polyp (arrow) and pedicle (arrowhead) shown by CFCUS.
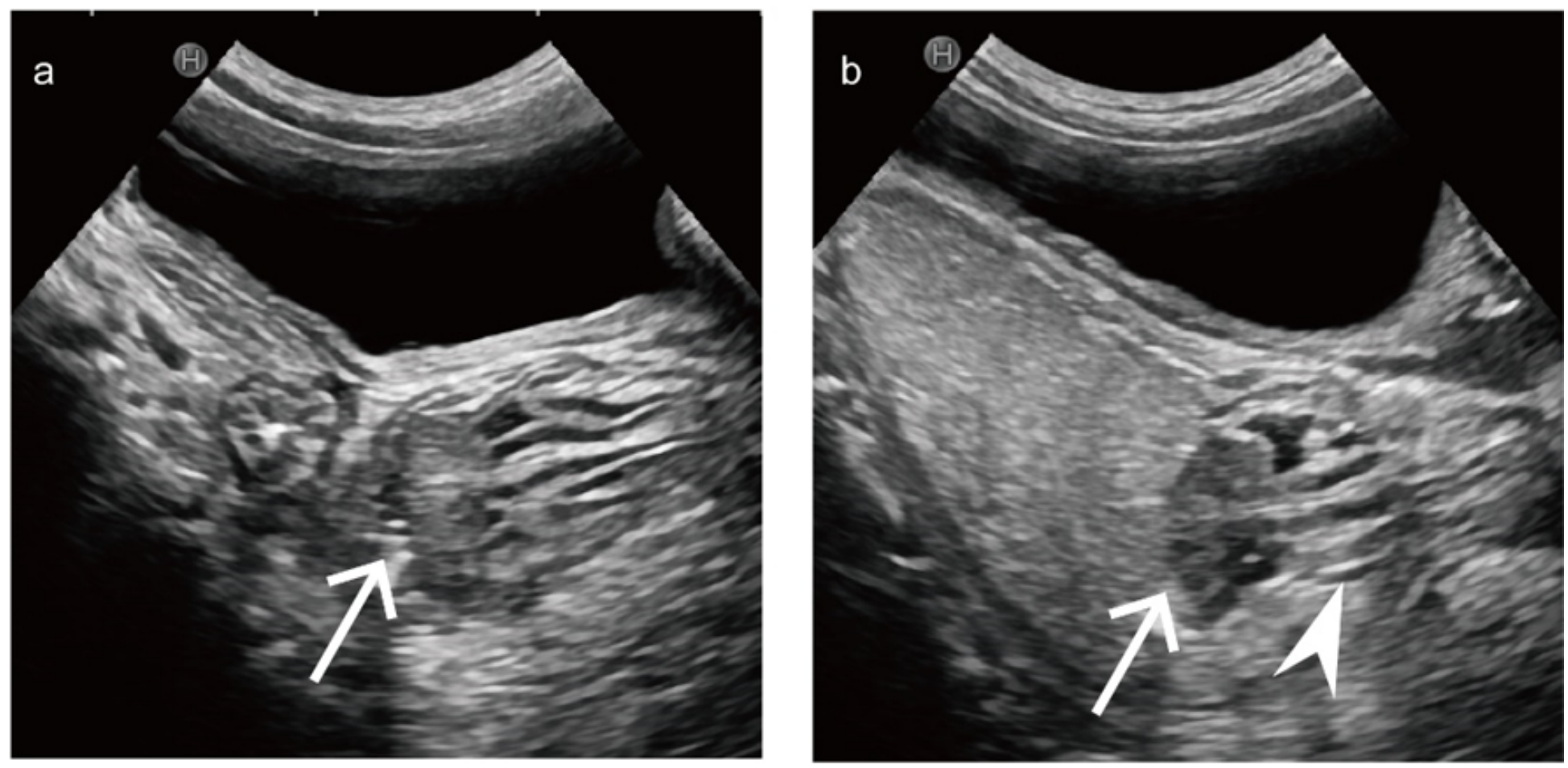

\section{Figure 5}

A 7-year-old boy. (a) CTUS showed a small uniform medium echo (arrow) with an unclear boundary. (b) CFCUS revealed a typical sonographic appearance of the polyp: intraluminal uniform medium echoes and ovoid hypoechoic nodules (arrow), with a definite boundary, associated with a rough pedicle (arrowhead) connected to the polyp, forming a typical "mushroom" sign. 

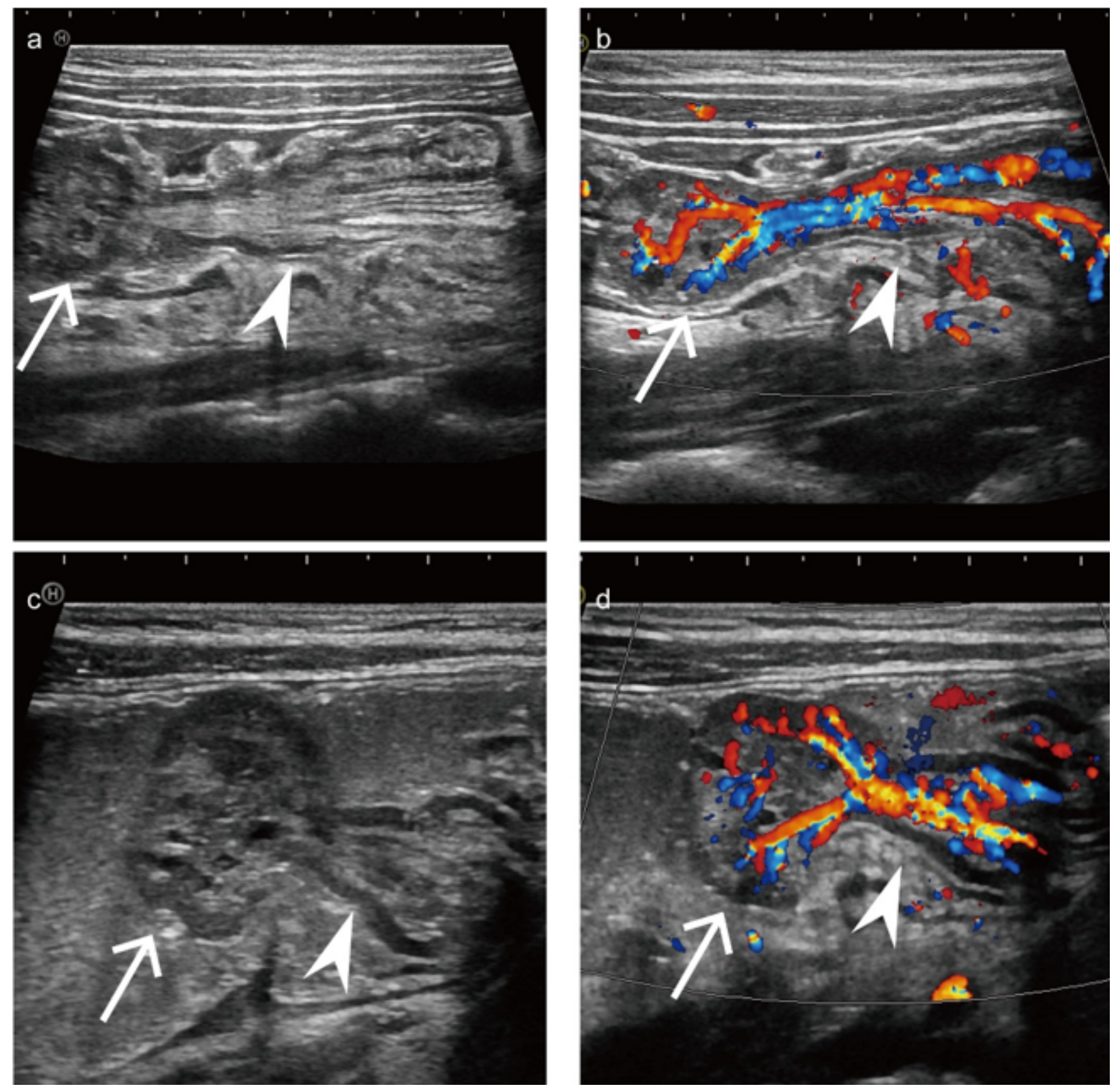

Figure 6

A 4-year-old girl presented to the hospital for painless rectal bleeding for 5 days. (a-b) CTUS showed a polyp (arrow) with an internal cyst cavity, and the length of the pedicle (arrowhead) was overestimated. Colour Doppler revealed abundant blood flow signals within the polyp. (c-d) The exact length of the pedicle (arrowhead) in the polyp (arrow) was identified by CFCUS. 

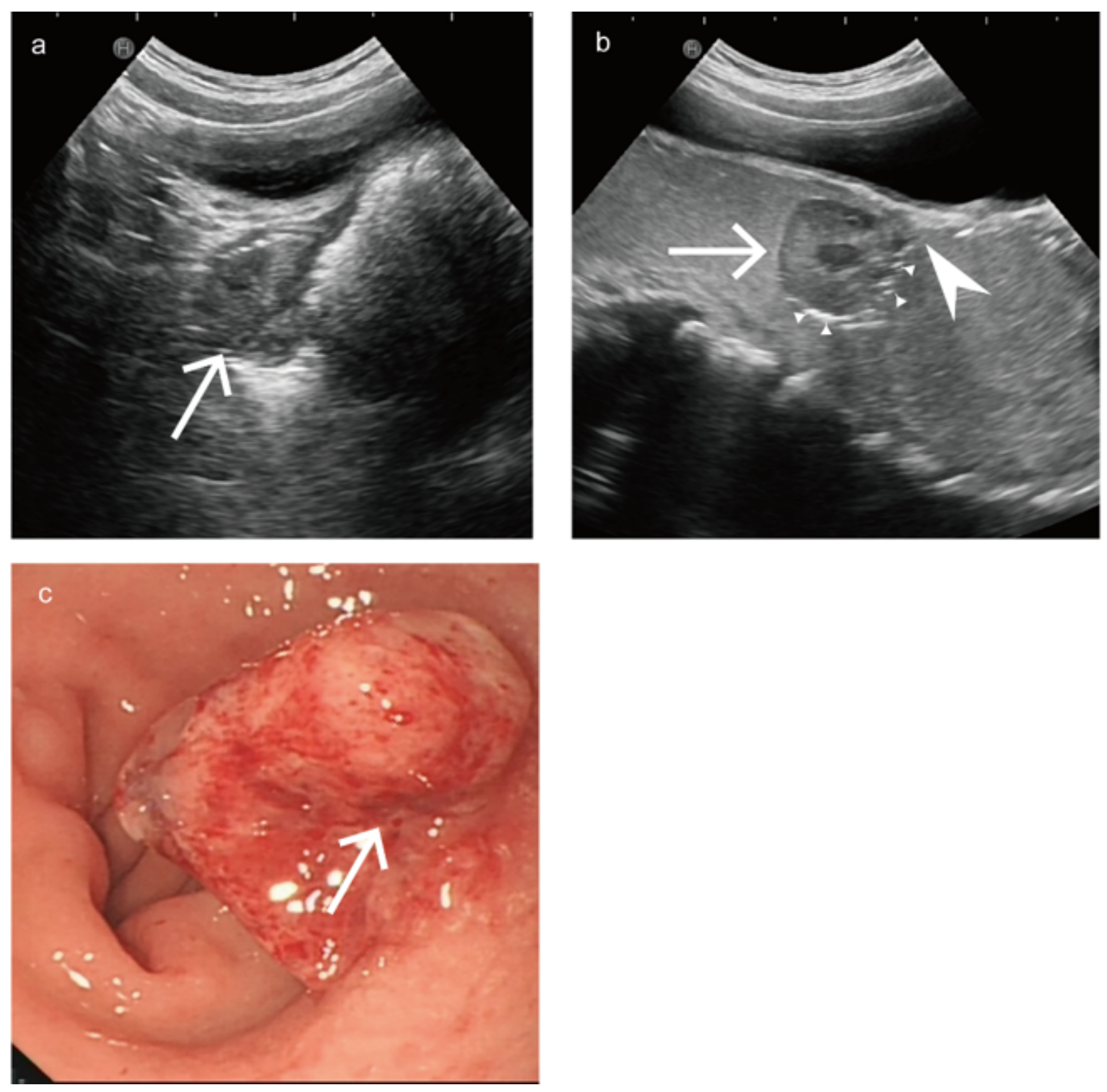

\section{Figure 7}

A 3-year-old girl was admitted to our hospital for painless rectal bleeding. (a) CTUS image of the polyp (arrow) without adequate bowel preparation. (b) The colonic polyp with a "flat" pedicle (arrowhead) was accompanied by punctate hyperechoicity (triangle arrow) on the surface by CFCUS. (c) Colonoscopy image showing a Yamada type III polyp (arrow), with erosion and active bleeding on the surface. 


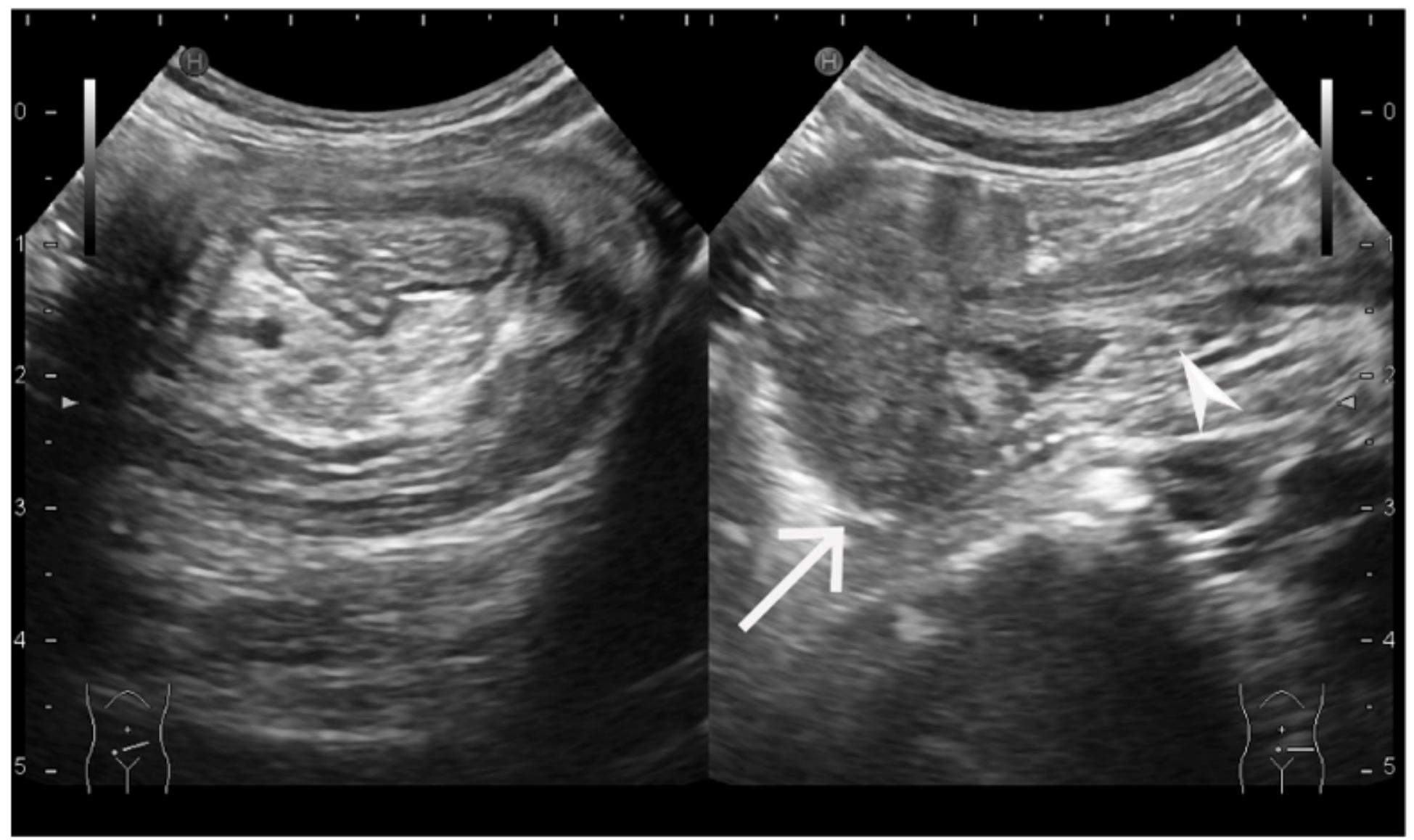

Figure 8

A 5-year-old girl with a juvenile polyp presented to the emergency department after a sudden onset of abdominal pain for $5 \mathrm{~h}$. On the left, the transverse image of the ultrasound indicates multiple concentric circles, demonstrating intussusception. The right image shows a hypoechoic nodule suggestive of a polyp (arrows), and a pedicle (arrowhead) was observed in the intussusceptum. 